\title{
Review of: "Automated recognition of the cricket batting backlift technique in video footage using deep learning architectures"
}

Anilkumar K K ${ }^{1}$

1 Cochin University of Science and Technology

Potential competing interests: The author(s) declared that no potential competing interests exist.

- Positive aspects of the study:

1. Application of deep learning using transfer learning technique to a new area which is very promising.

2. Identification of cricket batting backlift technique is not seemed to be attempted earlier using machine learning or deep learning.

3. Achieved higher classification accuracies of the order of $98 \%$ and above.

4. The efficacy comparison results of the various CNNs can be used by researchers to select appropriate deep learning networks.

5. The dataset can be used by other researchers in this area.

- Drawbacks:

1. The study explains the architecture of various pretrained networks used in detail which is unnecessary as these details are publicly available.

2. The dataset used is small for deep learning.

3. The values of hyperparameters used is not given. 